\title{
Performance Examination of OFDM Modulation Techniques in LTE 4G
}

\author{
J. Manikandan, V. Mangaiyarkarasi, P.Subramanian
}

\begin{abstract}
The digital modulation methods are being chosen in high data rate systems as Long Term Evolution (LTE) and LTE-A. Quadrature Phase Shift Keying (QPSK) and Binary Phase Shift Keying (BPSK) is the simplest form of the PSK with double carrying capacity when compare to the other traditional techniques in modulation. In conventional method the performance was analyzed in MIMO .In this paper proposed the performance analysis of BPSK and QPSK modulator and demodulator in LTE 4G system models under the Additive white Gaussian Noise (AWGN) and Rayleigh fading by comparing the Bit Error Rate (BER). From the analysis, compare to BPSK QPSK has good BER. Using the MATLAB Simulink tool Implementation is performed.
\end{abstract}

Keywords: LTE, BPSK, QPSK, simulink.

\section{INTRODUCTION} developed by 3GPP and first commercialized by Telia Sonera in 2009 referred as as 4G LTE [8]. LTE is the development of the UMTS/ GSM standards. The objectives of LTE was to enlarge the speed as well as capacity of wireless data networks using DSP technique, redesign and simplification of the network structural design, offers enhanced data rate, power utilization, latency, cell edge throughput etc. LTE presents lesser data transfer latencies i.e. in most advantageous situation with small IP packets latency is sub-5 ms, LTE have lesser latencies for link setup time and handover time then traditional radio access technology. Realization easiness and robustness is done by BPSK in the application of satellite communication. When compare to the BASK, BPSK has enhancement in $3 \mathrm{~dB}$ power as well as capacity of carrying double data information. The phase and frequency of the regenerated carrier wave must be synchronized with the carrier wave used on the transmitting end. QPSK allows the signal to carry twice as much as information as ordinary PSK using the same bandwidth. QPSK is utilized in the applications of satellite transmission namely mobile phones, video conferencing, and various digital communications above RF carrier

Revised Manuscript Received on December 30, 2019.

* Correspondence Author

J.Manikandan*, Associate Professor,ECE,Sri Sairam Engineering College,Chennai-44,India. E-mail: manikandan.mangai@gmail.com

V.Mangaiyarkarasi, Assistant Professor ,EIE, Valliammai Engineering College, Chennai, India. E-mail: mangai.manikandan@gmail.com

P.Subramaninan, Professor, Sri Indu College of Engineering \& Technology, Hyderabad. Email: 69subbu@gmail.com

(c) The Authors. Published by Blue Eyes Intelligence Engineering and Sciences Publication (BEIESP). This is an open access article under the CC BY-NC-ND license (http://creativecommons.org/licenses/by-nc-nd/4.0/)
LTE is Wireless data communication standard, and was

The main objectives of this work is to show the performance analysis of the BPSK and QPSK in terms BER including less cost and capability to offer enhanced data rates in LTE. Further the paper are organized with the literature study in section 2 proposed method in section 3,results and discussions in section 4 and conclusion in section 5 .

\section{LITERATURE STUDY}

Author Sharma, S. and Singh, H [1] Comparison of various modulation schemes used in the OFDM systems with least bit rate and high data rates. Navita ,A , Shanmugasundaram T.A. [2-3] analyzed the performance of the MIMO,SC-FDMA and OFDMA in LTE at physical layer by considering various modulation designs namely BFSK, QPSK,BPSK,16QAM by utilizing SNA as well as BER as parameters.

Yellampalli, S [3] modeled the Costas loop to the memory. Also compares the folded and unfolded filter realizations and implemented using MATLAB and Simulink. This method finds out best approach to realize a Costas loop receiver. Bushnaq, S et al [4] presented the digital wireless transceiver using proposed method for data enhancement. But the ranges limits less than $2 \mathrm{~cm}$.Also this transceiver uses in ISM by using the power amplifier in the transmitter side and LNA with receiver side but it occupies large power consumption.

Miyauchi, T., et al [5] designed the high speed programmable SISO decoder LSI for decoding turbo codes. On the basis of the original memory architecture SW-Log-BCJR algorithms is realized. Form this method it attains high operating frequency with less coding loss. Belkacem, Y.M. and Bentata, S.E [6] performed the BPSK signal demodulation through DSP algorithms namely matching filters to minimize the symbol timing ISI effect and the received signal is examined with respect to CCSDS standards and reference signal.

Manikandan. J and Dr. M. Manikandan [7] realized basic modulation as well as demodulation principles of BPSK and QAM for OFDM applications. The comparison analysis of power, speed and area were shown between the QAM and BPSK. It simulated using Modelsim 6.3c and synthesized using Xilinx 12.4i.Treuttel, J et al [9] demonstrated to produce modulated signals of QPSK with the help of multiplier. Khanna, et al [10]-[12] Proposed the module of QPSK on the basis of $\pi / 4$ modulation and the bandwidth efficiency were calculated and also compare with the QPSK address. 


\section{Performance Examination of OFDM Modulation Techniques In LTE 4G}

\section{PROPOSED METHOD}

To represent binary symbol of either 0 or 1 , one of the two state signals are shifted from the phase waveform respectively. The carrier equation is expressed as

$$
S(t)=A \cos \left(2 \pi f_{c} t\right)
$$

Where, A is the peak amplitude of the sinusoidal. Coherent BPSK has single dimensional signal space with two message points, in order to produce a BPSK signal, the binary input data in polar form with symbol 1 and 0 are characterized with a constant amplitude level of $\sqrt{E_{b}}$ and $-\sqrt{E_{b}}$. The signal transmission encoding method is passed out by a Non Return Zero level encoder (NRZ). The binary output wave together with the sinusoidal carrier $\boldsymbol{\Phi} 1(\mathrm{t})$, whose frequency is given by $f_{c}=\frac{n_{c}}{T_{b}}$ are given into the product modulator as well as the desired BPSK wave is obtained as the modulator output. Other merit of BPSK when compared to BASK is 3dB power enhancement and double information carrying capacity. Though it is significant to note i.e. modulation technique is bandwidth ineffective due to the fact that it is only able to transmit 1bit/symbol, thus creating it inapt for the application of high speed data rate. The numerical illustration for BPSK signal is written as

$$
S c(t)=\frac{\sqrt{2 E_{b}}}{\sqrt{T_{b}}} \cos (2 \pi f c t)+\pi(1-n) \ldots . n=0,1
$$

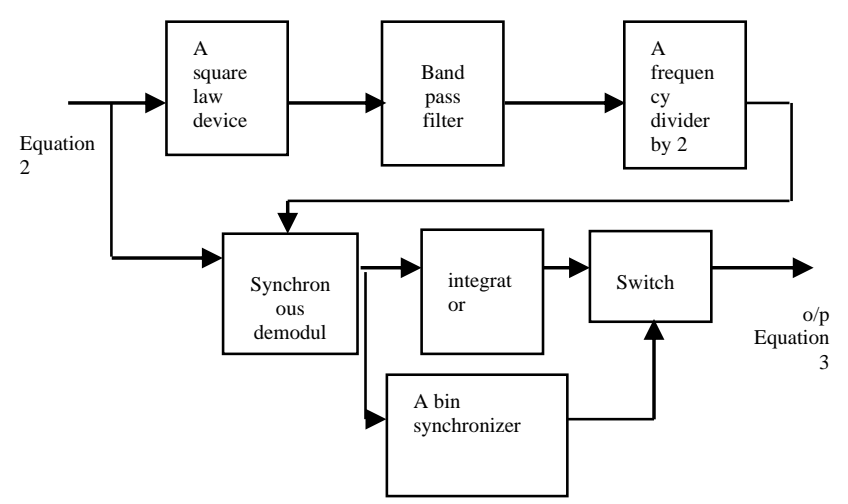

Fig. 1.Block diagram for equation generation of BPSK.

If In the above figure 1 the equation 2 is given as the input signal to the square law device and the its output is represented as $\operatorname{Cos} 2\left(2 \pi f_{c} t+\theta\right)$.Then the output of the square law is given to the band pass filter and it produce the output as $\operatorname{Cos} 2(2 \pi f c t+\theta)$.This output is given to the synchronous demodulator and it represent $b(t) \sqrt{2 p} \cos 2(2 \pi f c t+\theta)$ and it gets integrator with bin synchronizer and according to the switch the output signal will represented as

$$
S o(K T b)=b(k T b) \sqrt{p / 2 T b}
$$

BPSK system under constant noise level, transmitted bit energy and other distortion provides the smallest BER because BPSK method has the highest space between two signal ends. The BER of BPSK in AWGN can be obtained using

$$
P e, B P S K=V \sqrt{\frac{2 E_{b}}{N_{0}}}
$$

Where, Eb is energy per bit and N0 is the noise power spectral density. $V$ is the $Q$ function which is utilized commonly for computing the area under the tail of the Gaussian pdf which is represented by $V(i)$.

$$
V(i)=\frac{1}{2 \pi} \int_{x}^{\infty} e^{-\left(\frac{t^{2}}{2}\right)} d t
$$

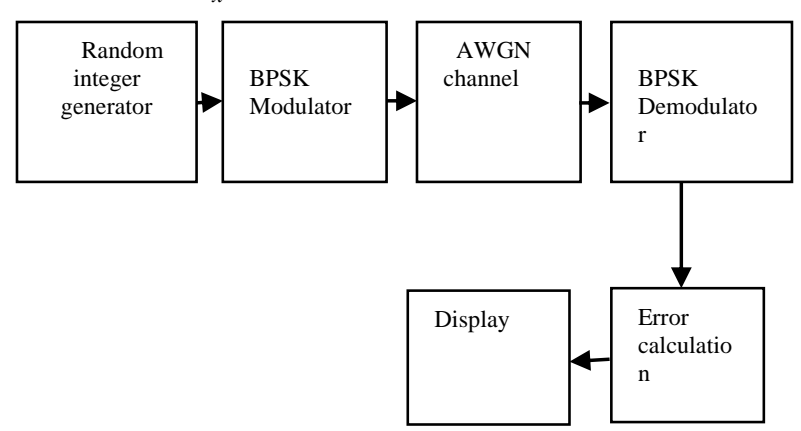

Fig. 2.Block of BPSK in MATLAB Simulink.

Figure 2 shows the BPSK modulator and demodulator block in MATLAB Simulink using AWGN channel and Random integer generator. QPSK doesn't represent in 1 or obut it represent 001011 or 01.In QPSK two sinusoids are taken in the modulation as well as demodulation basis. One symbol contains two bits in QPSK modulation. The equation represented for QPSK is represented as

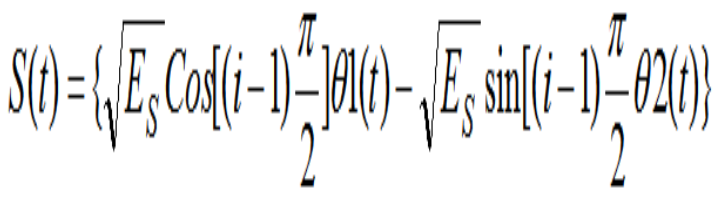

Where $\mathrm{i}=1,2$, 3, 4 Es-energy per symbol, Ts -symbol period, -reference signal. Compare to the BPSK the QPSK is more bandwidth efficient

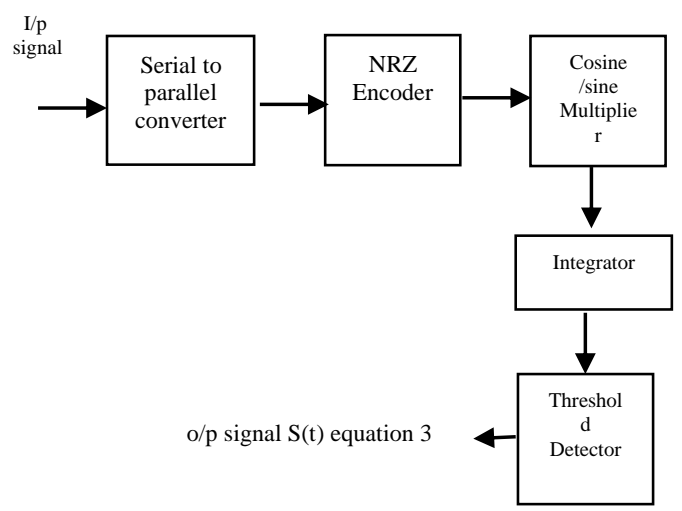

Fig. 3.Block diagram for Equation generation of QPSK.

Before The bit error rate equation for QPSK is written as

$$
P_{e}=V\left[\sqrt{\frac{2 E_{b}}{N_{0}}}\right]
$$

The above figure 3 illustrates QPSK equation representation the given input signal is converted to serial to parallel and it gets encoded by NRZ with even and odd the even code is multiplied with the cosine value and odd code is gets multiplied with the sine value and this reference value is get integrator and the output signal $s(t)$ is detected using threshold detector

Published By:

Blue Eyes Intelligence Engineering

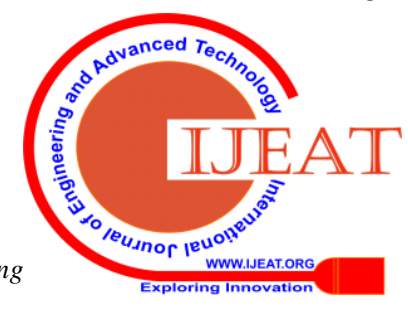




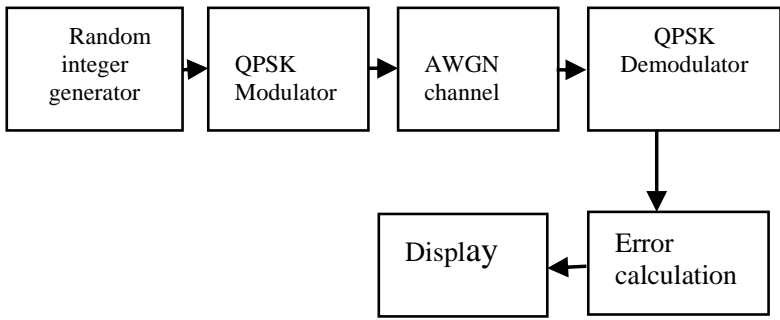

Fig. 4.Block diagram of QPSK in MATLAB Simulink

Figure 4 shows the QPSK block in MATLAB Simulink using AWGN channel and random integer generator.

\section{RESULT AND DISCUSSION}

The performances were analyzed using MATLAB Simulink. Figure 5 shows the BPSK modulator and demodulator design. Similarly Figure7 shows the design of QPSK modulator and demodulator.

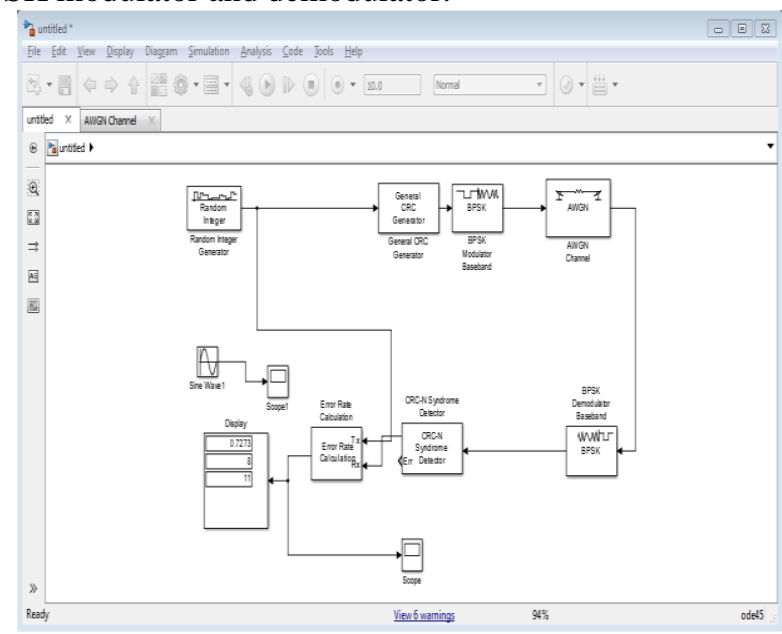

Fig. 5.Design of BPSK modulator and demodulator.

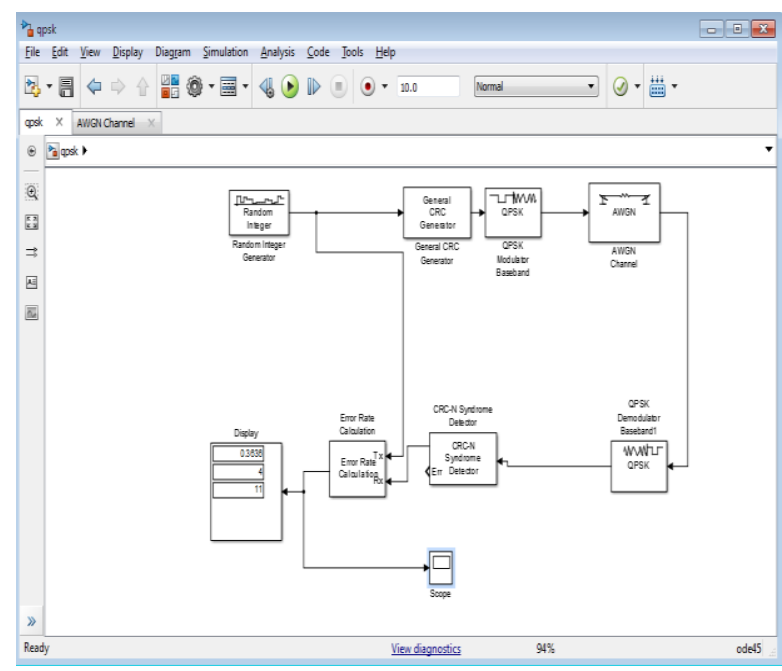

Fig. 6.Design of QPSK modulator and demodulator.

\section{CONCLUSION}

In this paper describes the performance evaluation of the digital modulation in LTE 4G applications for BPSK and QPSK techniques. The performance in BPSK and QPSK has been simulated using MATLAB Simulink. BER is employed as main factor in differentiating the performance of data channels. Form this analysis the QPSK has good BER compare to the BPSK in the application of LTE 4G.

\section{REFERENCES}

1. S. Sharma, and H. Singh, "Comparison of Different Digital Modulation Techniques in LTE System using OFDM AWGN Channel: A Review", International Journal of Computer Applications,Vol.3,No.3,Jun.2016,pp.1-4.

2. A. Navita, "Performance analysis of OFDMA, MIMO and SC-FDMA technology in 4G LTE networks" International Conference Cloud System. Big Data Engineering, Jan.2009, pp. 554-558.

3. S. Yellampalli, "A low-area based costas loop implementation for BPSK signals", IEEE International Conference on Electrical, Electronics, Communication, Computer, and Optimization Techniques, Dec.2017,pp. 196-200.

4. T.A. Shanmugasundaram. and V. Vijayabaskar, "Bit error rate analysis of RS $(7,3)$ coded frequency shift keying using simulink", American Journal of Applied Sciences, Vol. 12, No. 2, 2015, pp.92-98.

5. S. Bushnaq, T. Nakura, M. Ikeda, and K. Asada, "All digital wireless transceiver using modified BPSK and 2/3 sub-sampling technique". IEEE International Conference on ASIC, Oct. 2009,pp. 469-472.

6. T. Miyauchi, K. Yamamoto, T. Yokokawa, M. Kan, Y. Mizutani, and M. Hattori," High-performance programmable SISO decoder VLSI implementation for decoding turbo codes", IEEE Global Telecommunications Conference, Vol. 1, Nov. 2001, pp. 305-309.

7. Y. M. Belkacem, and S. E. Bentata, "BPSK Demodulator Signal Processing for Satellite Communication System", IEEE International Conference on Recent Advances in Space Technologies, Jun. 2019, pp. 485-490.

8. J. Manikandan. and M. Manikandan, "Optimized BPSK And QAM Techniques For OFDM Systems". International Journal of Computer and Technology ,Vol.9,No.6.

9. M. A. N. Sukar, and M. Pal," SC-FDMA \& OFDMA in LTE physical layer", International Journal of Engineering Trends and Technology, Jun.2014,Vol.12,No.2, pp.74-85.

10. J. Treuttel, A. Maestrini, J. Sarrazin, and F. Joint," $330 \mathrm{GHz}$ and 600 $\mathrm{GHz}$ Schottky Heterodyne Systems for QPSK Terahertz Telecommunication", IEEE International Conference on Telecommunications, Jun.2018, pp. 291-294.

11. A. Khanna, A. Jaiswal, and H. Jain, "Design and synthesis of bandwidth efficient QPSK modulator for low power VLSI design", IEEE International Conference on Electronics and Communication Systems pp. 1235-1240.

12. C. U. Ndujiuba, O. Oni and A. E. Ibhaze, "Comparative analysis of digital modulation techniques in LTE 4G systems". Journal of wireless Networking and Communications, Aug.2015,Vol.5,No.2, pp.60-66.

\section{AUTHORS PROFILE}

Dr. J.Manikandan, Ph.D, Associate Professor at Sri Sairam Engineering College in the Department of Electronics \& Communication Engineering. His research activity mainly in the field of Radio

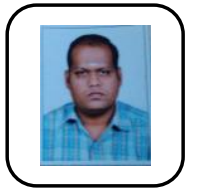
frequency and Microelectronics. He has published more than 15 papers in journals or conferences. He is a member of the Institution of Electronics and Telecommunication Engineers Society.

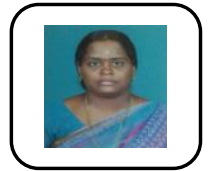

Mrs.V. Mangaiyarkarasi, Received Masters Degree In Process Control And Instrumentation From Annamala University, Tamilnadu, India In 2005. Since 2008 She Has Been An Assistant Professor At SRM Valliammai Engineering College, India Teaching Courses In Instrumentation And Signal Processing. She Published More Than 15 Papers In Journals And Conferences. Her Research Interests Include Signal Processing And Process Control. She Is A Member In ISTE And ISC Chapters.

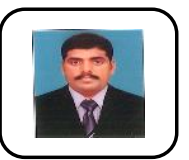

Dr. P.Subramanian, Received His MCA Degree From Bharathidasan University, Trichy, India In 2002 And M.Tech With Computer Science And Engineering From SRM University, Chennai, India In 2007. He Received The Ph.D, Degree From St. Peter's University, Chennai, India In 2016. From 2003 To 2008, He Worked With Valliammai Polytechnic College As A Lecturer.

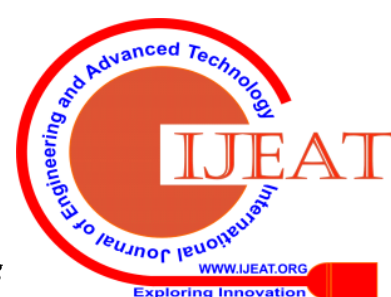


Performance Examination of OFDM Modulation Techniques In LTE 4G

From 2008 To 2016 He Worked With Shri Andal Alagar College Of Engineering As Associate Professor During The Time Of Relieving. From 2016 To 2018 He Worked With Guru Nanak Institute Of Technology, Hyderabad As Professor And Head For IT Department. Since 2018 He Has Been With Sri Indu College Of Engineering \& Technology, Hyderabad As Professor. 